\title{
La autoevaluación como actividad docente en entornos virtuales de aprendizaje/enseñanza
}

\author{
Ángel García-Beltrán \\ Universidad Politécnica de Madrid, Madrid, España \\ Raquel Martínez \\ Universidad Politécnica de Madrid, Madrid, España \\ José-Alberto Jaén \\ Universidad Politécnica de Madrid, Madrid, España \\ Santiago Tapia \\ Universidad Politécnica de Madrid, Madrid, España
}

Self-assessment in virtual teaching and learning environments

\begin{abstract}
Resumen
En este trabajo se describen las diferentes actividades docentes que pueden realizarse con los sistemas de autoevaluación del aprendizaje que están incluidas en muchas de los actuales entornos virtuales basados en el servicio web. Junto a cada una de ellas se indican algunas experiencias reales llevadas a cabo por los propios autores y otros profesores de la Escuela Técnica Superior de Ingenieros Industriales en actividades docentes de asignaturas pertenecientes a titulaciones oficiales impartidas en la ETSII-UPM. El entorno virtual utilizado en estas experiencias es la plataforma AulaWeb.
\end{abstract}

Palabras clave: Autoevaluación, prueba objetiva, teleformación, aprendizaje mixto, entorno virtual

\begin{abstract}
In this work, several activities that can be carried out by means of self-assessment modules of web-based learning management systems are described. Some self-assessment experiences developed by the authors and other teachers of the ETSI Industriales of the Universidad Politécnica de Madrid in activities of different courses taught in this engineering faculty are explained. The learning management system used in these experiences is AulaWeb.
\end{abstract}

Keywords: Self-assessment, objective test, e-learning, blended learning, new technologies, virtual environment

\section{Introducción}

Desde el punto de vista didáctico, la evaluación o control como elemento de verificación es concepto crítico en el aprendizaje y un pilar fundamental que debe 
permitir medir y valorar la bondad del proceso de aprendizaje considerado globalmente, tanto de la programación como de la puesta en práctica del mismo. La no consecución de los objetivos planteados a priori debe implicar la ineficacia del proceso tal como se ha diseñado o llevado a la práctica, y la necesidad de reestructurarlo en mayor o menor medida con el objetivo de mejorarlo (Villar, 2004). Por lo tanto, la evaluación del aprendizaje cumple básicamente dos funciones fundamentales: permite el ajuste pedagógico a las características individuales de los alumnos y determina el grado de consecución de los objetivos previstos (Serrano, 2004).

Entre las principales técnicas de evaluación del aprendizaje cabe destacar los exámenes de teoría, los exámenes de problemas, los exámenes orales, la realización de trabajos y proyectos y las pruebas de respuesta objetiva o de tipo test (Bloom, 1975). Seguidamente se describen y comentan estos métodos y técnicas de evaluación, señalando las ventajas y desventajas más importantes de cada uno de ellos.

Los exámenes de teoría se orientan a valorar la cantidad de información retenida por los alumnos. Entre las ventajas de este tipo de examen destacan la relativa facilidad de preparación, adecuación cuando el número de estudiantes es grande, la posibilidad de evaluación del trabajo individual y del nivel de conocimientos adquirido, la obligación por parte del alumno a organizar la respuesta permitiendo juzgar además de la extensión de los conocimientos, la capacidad de selección y síntesis del examinado tanto en el desarrollo de la prueba como en el estudio y, finalmente, el reducido tiempo de realización ya que todos los alumnos se examinan simultáneamente. Entre los inconvenientes se subrayan que puede convertirse en una prueba basada en planteamientos memorísticos, la posibilidad de que no sea representativa la asignatura por problema de acotación de la extensión del examen y la no uniformidad/objetividad en los criterios de corrección por parte del profesor.

Los exámenes de problemas permiten valorar la capacidad de relación y de razonamiento de los alumnos. Este tipo de exámenes permite también evaluar mejor el trabajo realizado por el alumno a lo largo del curso, lo que es fundamental en este tipo de asignatura de perfil práctico. En algunas áreas de conocimiento, el principal problema puede estribar en la dificultad de diseñar el enunciado y corregir las respuestas. Por ejemplo, en el caso de la enseñanza de lenguajes de programación de ordenadores se pueden preparar problemas en los que se pida como resultado la codificación de un programa o una parte de él que cumpla una serie de requisitos muy precisos y lleve a cabo una tarea determinada.

Los exámenes orales permiten el mayor grado de libertad posible al alumno en la respuesta, ya que normalmente, se le pide que desarrolle por completo, durante un tiempo determinado, uno o varios temas teóricos, o bien que resuelva uno o varios problemas concretos. Sus principales ventajas: es la forma de examen más flexible ya que las preguntas pueden adaptarse al propio desarrollo de la prueba, lo que permite obtener la visión más amplia y completa de los conocimientos del estudiante, aumenta la interacción entre el alumno y el profesor, minimizando la posibilidad de error en la interpretación de la pregunta, permite observar la capacidad de respuesta del alumno ante situaciones concretas, semejantes a las que posiblemente tendrá que resolver durante su vida profesional y obligan a pensar, razonar y organizar la respuesta sobre la marcha, permitiendo juzgar la capacidad de improvisación del examinando. El principal inconveniente es la gran cantidad de tiempo que ocupan, por lo que en las condiciones 
masificadas de los centros actuales (especialmente los universitarios), son casi irrealizables. Normalmente se reserva para casos especiales o grupos con un número muy reducido de alumnos.

La realización de trabajos o proyectos puede convertirse en otra forma de evaluación consistente en el diseño y desarrollo de un trabajo o proyecto que puede entregarse durante o al final de la docencia de la asignatura. Este tipo de evaluación también puede implementarse en grupos con un número reducido de alumnos en el que cada uno de ellos se haga cargo de un proyecto o en grupos con un mayor número de alumnos que quede dividido en pequeños equipos, cada uno de los cuales se responsabilice de un proyecto. Este formato puede ser especialmente interesante para fomentar el trabajo en grupo de los alumnos. El principal inconveniente vuelve a ser el tiempo que la corrección de este tipo de trabajos le lleva al profesor, especialmente en grupos numerosos de alumnos.

Las pruebas de respuesta objetiva, cerrada o de tipo test tienen como una de sus principales ventajas el que se puedan diseñar para comprobar muchos tipos de objetivo. Sin embargo, algunos objetivos se escapan de las posibilidades de estas pruebas, pues el alumno no tiene oportunidad, por ejemplo, de expresarlas libremente, de organizar sus propias ideas o de hacer síntesis originales. Otra ventaja reside en su evaluación objetiva. Con las preguntas de respuesta abierta siempre cabe el riesgo de que el alumno responda correctamente pero no en la línea pretendida por el profesor para poder evaluar un objetivo determinado. Por otra parte, no siempre es fácil identificar en respuestas genuinamente correctas, pero amplias y divagantes, los datos específicos que permitan evaluar determinados objetivos. La versatilidad de las preguntas de respuesta objetiva constituye una ventaja que en ocasiones puede ser decisiva. El obstáculo inicial de este tipo de pruebas reside en la dificultad de su construcción. Su preparación puede requerir mucho tiempo e ingenio, de lo contrario es fácil hacerlas mal. Otro inconveniente reside en la facilidad para que los alumnos puedan copiarse las respuestas entre ellos durante la realización de la prueba en el aula.

De los cinco métodos de evaluación citados anteriormente sólo el último es implementado de forma sencilla, con corrección inmediata y automática (autoevaluación), en los entornos virtuales de aprendizaje/enseñanza. El motivo de esta restricción reside en la dificultad de corrección por parte de un ordenador de preguntas que no tengan una respuesta objetiva. También podría implementarse un sistema de entrega de trabajos y prácticas o de preguntas de repuesta abierta pero, en este caso sería necesario la actuación de un profesor para la tarea de corrección o de un software que incluya un corrector "inteligente". Esta última opción puede ser bastante costosa y difícil de desarrollar. Otros métodos como la presentación por videoconferencia o la evaluación de actividades de debate serían como mínimo igualmente complejos de autoevaluar.

Desgraciadamente, la prueba objetiva es una herramienta que se debe emplear en general a título informativo y que incluso el alumno puede realizar con carácter voluntario. El docente debe considerarla como una herramienta que puede mantener activos, motivados y orientados a los alumnos durante el proceso de aprendizaje. Por ejemplo, lo ideal sería realizar una prueba objetiva cada lección o cada mes. Este 
sistema regular de autoevaluación ayudaría a tener un conocimiento de la evolución de la asignatura tanto por parte del alumno como por parte del profesor.

Las principales ventajas en la utilización de un entorno virtual para llevar a cabo un sistema de autoevaluación con pruebas de respuesta objetiva son:

- Posibilita un seguimiento individualizado del aprendizaje del alumno.

- Permite evaluar conocimientos y habilidades.

- Facilita el establecimiento de una evaluación continuada durante el proceso de aprendizaje y reduce el tiempo de su diseño, distribución y desarrollo.

- Agrega una gran flexibilidad temporal y espacial del sistema tanto para la configuración de ejercicios como de su realización. En este sentido puede ser especialmente útil para permitir que el alumno pueda seguir su propio ritmo de aprendizaje.

- Proporciona una respuesta inmediata (retroalimentación) de los resultados de los ejercicios.

- El almacenamiento de los resultados facilita la creación de informes y tratamiento de datos tanto a nivel de un alumno o de un grupo de alumnos como de las preguntas utilizadas.

- La base de datos de preguntas puede reutilizarse en otros cursos.

- La no necesidad de corregir por parte del profesor lo hace especialmente apropiado para grandes grupos de alumnos.

\section{Implementación de sistemas de autoevaluación en entornos virtuales}

$\mathrm{Si}$, en general, los sistemas de evaluación deben adecuarse a los objetivos de aprendizaje, los contenidos y los destinatarios, si están implementados mediante un entorno virtual entonces deben adecuarse además a esta metodología específica (García, 2006). Un alto porcentaje de entornos virtuales de aprendizaje/enseñanza tienen desarrollado un sistema de pruebas de respuesta objetiva, de tipo test o autoevaluación mediante un módulo de software con acceso a una base de datos. Este tipo de sistema suele incluir entre sus funcionalidades la creación de preguntas para este tipo de pruebas, la configuración de ejercicios, la generación de las pruebas, la gestión y corrección de las respuestas y la gestión y almacenamiento de las respuestas... la potencia y flexibilidad del sistema dependerá de las características de cada una de estas facilidades.

En el ámbito de las preguntas que componen las pruebas es importante destacar la variedad de tipos de cuestiones. Entre los tipos de preguntas de corrección automática se pueden encontrar cuestiones de verdadero-falso, de tipo test de respuesta simple, de tipo test de respuesta múltiple, ejercicios con solución numérica entera o real (incluyendo una tolerancia en la respuesta), preguntas de relación o emparejamiento, preguntas de ordenación, de rellenar huecos, ejercicios de respuesta corta o de cadena de caracteres, ejercicios cuya solución es proporcionada de forma gráfica (por ejemplo, puzzles o cronogramas), tablas, mapas de imágenes... También es necesario considerar la posibilidad de incluir elementos multimedia en el enunciado: imágenes, gráficas, ecuaciones, sonidos, vídeos... 
En el terreno de la generación de las pruebas debe considerarse la capacidad de crear los ejercicios a partir de una base de datos de preguntas, la flexibilidad en el diseño de pruebas de evaluación con capacidad para controlar aspectos tales como: puntuación, número de intentos, duración de la prueba..., así como la posibilidad de crear puertas de acceso condicionado a su superación, la variedad de plantillas disponibles para generar e interconectar ejercicios de evaluación o la posibilidad de integrar el uso de emuladores o simuladores como parte de la evaluación que se va a efectuar, ....

La mayoría de los entornos virtuales de aprendizaje/enseñanza más utilizados en la actualidad, como WebCT (http://www.webct.com/), Moodle (http://www.moodle.org/) o Claroline (http://www.claroline.net), disponen de algún módulo o sistema de autoevaluación con pruebas de respuesta objetiva. Puede encontrarse una lista más completa de entornos virtuales y sus características en el servidor Web del Gabinete de Tele-educación de la Universidad Politécnica de Madrid (http://www.gate.upm.es/).

Como ejemplos de herramientas de creación de ejercicios con preguntas de respuesta objetiva fuera de entornos virtuales de aprendizaje/enseñanza pueden destacarse:

Hot Potatoes (http://hotpot.uvic.ca/),

Puzzlemaker (http://puzzlemaker.school.discovery.com/),

Clic (http://clic.xtec.es/es/index.htm),

QuestionMark (http://www.questionmark.com/home.htm),

Quizmaker (http://www.quizmaker.com/) y

TestPilot (http://www.clearlearning.com/).

\section{El módulo de autoevaluación del entorno AulaWeb}

AulaWeb es una plataforma de tele-enseñanza desarrollada en el Departamento de Automática, Ingeniería Electrónica e Informática Industrial de la ETSII-UPM que se ha utilizado fundamentalmente como herramienta de apoyo para la impartición y el seguimiento de asignaturas presenciales de las carreras impartidas en sus centros (blearning) pero también como herramienta de e-learning de cursos a distancia. La aplicación fue inicialmente concebida para facilitar el aprendizaje a los alumnos que por primera vez recibían conocimientos de informática como asignatura presencial en la ETSII-UPM (Martínez, 2001)

El entorno de la aplicación permite la realización de varias actividades docentes mediante el empleo de un ordenador con conexión a Internet y un navegador web independientemente de su sistema operativo. La interfaz de AulaWeb está basada en una estructura mixta de iconos y menús, que permite al usuario saber en todo momento como acceder a una determinada zona o módulo del sistema con el objeto de facilitar al máximo la navegación del usuario. La aplicación incluye un sistema de seguridad a nivel de usuario para el acceso de alumnos, profesores y administrador general que se realiza a través de la red y tres interfaces distintas según el tipo de usuario: alumno, profesor y administrador general. Mediante un entorno amigable y sencillo de utilizar, se trata de facilitar tanto al alumnado como al profesorado, tareas como la publicación y recogida de información y recursos formativos, la realización y entrega de trabajos y prácticas, la ejecución de actividades de autoevaluación o el establecimiento de tutorías telemáticas en tiempo real. 
El módulo de autoevaluación de AulaWeb incluye varias funcionalidades (GarcíaBeltrán, 2002):

1) un gestor de preguntas que facilita al profesor la introducción de preguntas en la base de datos de la aplicación. Las preguntas pueden ser distintos tipos: verdadero-falso, test de selección simple, test de selección múltiple, de respuesta numérica entera o real, de respuesta de tipo cadena de caracteres, de enunciado variable y de respuesta tipo código de programación en TurboPascal. Además, el profesor puede asignar a cada pregunta un capítulo del temario y un nivel de dificultad a priori (muy fácil, fácil, medio, difícil y muy difícil).

2) un sistema de configuración que permite indicar todos sus parámetros de los ejercicios: título del ejercicio, grupo de alumnos al que va dirigido, número de preguntas, tipo de preguntas (fijas o aleatorias y de test, numéricas, de programación...), capítulo y nivel de dificultad de las preguntas, tiempo límite (duración en pruebas cronometradas), forma de corrección, fecha límite de finalización y máscara de red de los ordenadores conectados a internet desde los cuales se puede realizar el ejercicio.

3) el generador y administrador de ejercicios que, en base al contenido de la base de datos de preguntas y a los parámetros de configuración del ejercicio correspondiente, compone su contenido, presenta las preguntas y almacena las respuestas y los resultados en la base de datos y muestra dichos resultados a alumnos y profesores de la asignatura correspondiente.

\section{Casos de uso de la autoevaluación como actividad docente}

En este apartado se indican diferentes casos en las que se ha utilizado un sistema de autoevaluación del entorno virtual AulaWeb en actividades docentes antes, durante y después del periodo académico en prácticas o asignaturas pertenecientes a titulaciones oficiales impartidas en la Escuela Técnica Superior de Ingenieros Industriales de la Universidad Politécnica de Madrid. Si bien el objetivo común es el de promover el aprendizaje, cada caso tiene un conjunto distinto de condiciones y se han planificado y diseñado detalladamente con vistas a los objetivos marcados en cada uno de ellos.

\section{Evaluación inicial y pruebas de nivel antes del comienzo del curso}

El análisis del perfil de conocimientos de los alumnos que van a iniciar un curso es fundamental para determinar las carencias iniciales de éstos y plantearse algunas preguntas acerca de la adecuación de las asignaturas y sus contenidos en función de dicho perfil. Esta información puede resultar crucial para que los responsables de la organización curricular y de las asignaturas puedan adaptar dinámicamente sus contenidos antes del comienzo del periodo docente. La siguiente cuestión que se plantea es cómo puede obtenerse información para realizar este análisis del perfil de conocimientos. Una posible solución a este problema sería la realización de una prueba común para todos los alumnos mediante un sistema de evaluación ad hoc basado en web de las materias que han estudiado el curso anterior y de cuyos contenidos, posteriormente, van a necesitar en el siguiente curso.

Como ejemplo de esta actividad se tiene el proyecto WebTutor que fue implantado desde 1995 y durante varios cursos para determinar el perfil de conocimientos de los alumnos de nuevo ingreso de la ETSI Industriales de la UPM (Coronado, 1998). En este 
proyecto varios centenares de alumnos eran evaluados de sus aptitudes en Matemáticas, Física, Química y Dibujo antes de comenzar el curso. Por un lado, los resultados individuales de los alumnos se facilitaban de forma automática e inmediata después de la prueba a cada uno de ellos y por otro, los resultados globales se suministraban a los responsables docentes del plan de estudios y de las asignaturas de primero. De esta forma, cada alumno podía conocer sus posibles carencias antes de comenzar el curso y los docentes podían adaptar los contenidos de las asignaturas antes del comienzo del curso.

\section{Evaluación continua}

La evaluación continua se implementa mediante un conjunto de pruebas, informes, trabajos, o controles sistemáticos realizados durante el periodo docente, utilizados parcial o totalmente para la evaluación del alumno. En general aporta información sobre el desarrollo del proceso de aprendizaje de forma paralela a la docencia (evaluación formativa): permite al propio alumno conocer el rendimiento de su trabajo y al profesor realizar el seguimiento del aprendizaje de un alumno o un grupo de alumnos. Además, este proceso puede evolucionar durante el curso, modificándose las necesidades de los alumnos y consecuentemente las adaptaciones a realizar en el soporte pedagógico (Villar, 2004). Los entornos virtuales basados en web facilitan esta tarea añadiéndole flexibilidad, inmediatez, automatismo y comodidad, especialmente con grupos numerosos de estudiantes.

Un primer ejemplo nos lleva a la utilización de la plataforma AulaWeb como sistema de autoevaluación continuada en diversas asignaturas presenciales impartidas por la Unidad Docente de Informática de la ETSII-UPM. En concreto, las asignaturas de Informática (asignatura troncal de primer curso), Programación en Java (asignatura de libre elección), Programación Orientada a Objetos (asignatura opcional de la titulación de $2^{\circ}$ ciclo de Ingeniero en Automática y Electrónica). Sólo en el caso de la asignatura de Informática, más de 600 alumnos se autoevalúan cada año casi semanalmente de los contenidos impartidos en las clases presenciales desde el curso 2000-01. La calificación media de los ejercicios permite subir hasta un punto de la calificación final de la asignatura (Arranz, 2006). A raíz de estas experiencias, el sistema se está utilizando actualmente otras asignaturas de los planes de estudios oficiales no del área informática como Química, Termodinámica o Economía.

Un segundo ejemplo se circunscribe en el área de cursos a distancia. En este formato se han realizado experiencias de utilización de la plataforma AulaWeb en varias asignaturas de libre elección (Java Programming, Information Technologies y Development of dynamic web pages) impartidas para alumnos de varias Universidades europeas durante los cursos 2004-05 y 2005-06. Como particularidad de estas asignaturas en las que no existían clases presenciales, el idioma empleado en las comunicaciones a través de chats, foros, correo electrónico, prácticas $\mathrm{y}$ autoevaluaciones ha sido el inglés y la calificación en los ejercicios de autoevaluación tienen un peso del $25 \%$ en la nota final de la asignatura. La realización de una autoevaluación continua se ha combinado con la publicación de los contenidos formativos siguiendo el estándar SCORM, lo que ha permitido un seguimiento completo del progreso en la asimilación de los contenidos de los cursos. 


\section{Evaluación combinada con clases teóricas}

La realización de ejercicios puede combinarse con la clase magistral con el objetivo de verificar la correcta asimilación de los contenidos tratados en la clase de forma inmediata y fomentar el aprendizaje constructivo del alumno. Como principal ventaja, este sistema permite al profesorado conocer el resultado del proceso de enseñanza/aprendizaje al momento. Los principales inconvenientes pueden ser el tiempo que es necesario dedicar dentro del horario de clases y la infraestructura necesaria para llevar a cabo la evaluación. En el caso de disponer o impartir las clases en un aula con ordenadores y la posibilidad de acceso a un entorno virtual, dichos inconvenientes puede quedar sensiblemente aminorados.

Como ejemplo de experiencia en este caso, desde el curso 2003-04 las clases de la asignatura de libre elección Programación en C siguen este formato: las clases imparten en un aula con un ordenador conectado a un cañón de proyección como herramienta de apoyo para la impartición de la clase para el profesor. En la misma aula se dispone de un ordenador con conexión a Internet por alumno para que todos puedan realizar simultáneamente las pruebas configuradas previamente por el profesor en el entorno virtual AulaWeb. En este caso el número de alumnos por aula y curso no supera la treintena, lo que hace factible que el profesor pueda mantener la atención y atender a las cuestiones y dudas que puedan surgir durante toda la actividad. Los resultados de los ejercicios realizados se contabilizan en la calificación final de la asignatura.

\section{Evaluación combinada con prácticas}

Como en la actividad anterior, en el caso de las prácticas, especialmente en cursos y asignaturas con un perfil científico-tecnológico, la evaluación antes o después de la realización de las prácticas puede ser especialmente interesante. La realización de una evaluación sobre el enunciado (entregado con suficiente antelación) de la práctica antes de su realización material obliga a los alumnos a una preparación previa mientras que si se realiza después permite evaluación el grado de asimilación de los conocimientos y/o habilidades incluidas en los objetivos marcados.

En este caso, cada una de las prácticas de la asignatura Regulación Automática I (asignatura obligatoria de tercer curso) se apoyan en la realización de una prueba de autoevaluación que verifica la asimilación de conceptos de las prácticas que se llevan a cabo durante todo el semestre (Ferre, 2006). Esta actividad se realiza en un aula con ordenadores situada junto al laboratorio de práctica donde se cita a los alumnos en grupos reducidos (máximo de 20 alumnos). La prueba de autoevaluación además de tener un determinado peso en la calificación de las prácticas, motiva a los alumnos en su esfuerzo y los orienta hacia los objetivos específicos de cada una de ellas.

\section{Evaluación final: exámenes}

Una vez finalizado el periodo académico la evaluación final determina el resultado de la valoración del grado en el que se conseguido los objetivos propuestos (evaluación sumativa). Un primer problema de esta actividad puede radicarse en la dificultad para la identificación segura del alumno que realiza la prueba. Un segundo inconveniente estriba en la conveniencia de incluir las mismas preguntas para todos los alumnos de 
una convocatoria de examen, de forma que nadie pueda considerarse injustamente evaluado con respecto a los demás. Estos dos inconvenientes pueden soslayarse si se realiza la prueba de forma presencial en un aula con ordenadores simultáneamente con todos los estudiantes. Para ello, es necesario que el tamaño del aula de ordenadores sea lo suficientemente grande para admitir a todo el grupo de alumnos.

Precisamente este esquema fue el utilizado para examinar durante las últimas convocatorias oficiales de examen a los alumnos matriculados en la asignatura Métodos Informáticos en Ingeniería perteneciente al antiguo Plan de Estudios (de 1976) de la titulación de Ingeniería Industrial. Los alumnos realizaban la prueba para la que disponían de un tiempo máximo de dos horas o dos horas y media (según la convocatoria) y, al finalizarla, recibían inmediatamente el resultado del examen junto con una copia en papel del enunciado, las repuestas y las calificaciones de cada una de las preguntas. El máximo de alumnos a examinar en una convocatoria fue de 35 (el aula empleada tenía un total de 68 puestos de ordenador).

\section{Conclusiones}

La implantación de la autoevaluación mediante entornos virtuales es perfectamente factible en pruebas de repuesta objetiva y permite la realización de distintas actividades docentes que promueven el aprendizaje antes, durante y después del periodo académico:

1. La evaluación inicial permite conocer el perfil de conocimientos inicial de los alumnos para decidir el contenido, orientación y nivel de las clases, así como el tipo de ayuda pedagógica.

2. La evaluación continua facilita información del avance en el proceso de aprendizaje de forma paralela a la docencia.

3. La evaluación combinada con las clases teóricas o prácticas permite medir in situ y de forma inmediata la asimilación de los contenidos docentes. En el caso de la evaluación previa a la realización de la práctica obliga a una actividad de estudio y asimilación individual del alumno, en muchos casos muy necesaria para los objetivos previstos.

4. La evaluación final permite valorar el grado de consecución de los objetivos docentes.

En todos los casos, el diseño y planificación de la autoevaluación debe ser coherente con los objetivos y el resto de la metodología docente a emplear. A diferencia de lo que ocurre con otras técnicas de evaluación, la ventaja de la retroalimentación inmediata en los sistemas de autoevaluación implementados con entornos virtuales constituye una clave fundamental en el proceso de aprendizaje, ejerce como elemento motivador para esfuerzo del alumno y le orienta eficazmente en sus actividades.

\section{Agradecimientos}

Los autores quieren agradecer el esfuerzo y dedicación de las personas que han colaborado en el proyecto de desarrollo de las distintas versiones del sistema AulaWeb: Aurora Alonso, José María Arranz, Pablo Avendaño, Marcos Aza, Juan Antonio Criado, Francisco de Ory, Miguel Fernández, Pilar García, Manuel González, Jorge Granado, Teresa Hernández, Isadora Iglesias, Antonio R. López, Diego López, Julio A. 
Martín, Francisco Mascato, David Molina, Luis Miguel Pabón, Juan Carlos Pérez, Alberto Valero, Eduardo Villalar y Carlos Zoido dentro de la División de Informática Industrial de la ETSII-UPM.

El trabajo de los autores ha sido parcialmente financiado por la DGES (Ministerio de Educación y Ciencia) en los proyectos de referencia SEJ2004-08035-C02-01 y SEJ2004-08004-C02-02.

Conclusión de la redacción original del artículo: Septiembre de 2006

García Beltrán, A. et al. (2016). La autoevaluación como actividad docente en entornos virtuales de aprendizaje/enseñanza. RED. Revista de Educación a Distancia, 50. Consultado (día/mes/año) en http://www.um.es/ead/red/50

\section{Referencias}

Amghar, Y., Martínez, R., Hassapis, G., Dolan, D. y Ward, A. (2006), Final Report eTutor project: Innovative e-learning methodology for tutors in multi-cultural, collaborative and synchronous context. Vol I and II, (European project: 20034718/001-001 EDU-LEARN). Disponible en red en la URL: http://www.if.insalyon.fr/intranet/etutor.

Arranz, J.M. García-Beltrán, A. Jaén, J.A. Martínez, R. y Tapia, S (2006), Utilización de AulaWeb como apoyo docente en las asignaturas de Informática en el curso 2005-06, 76 pp, marzo de 2006. Disponible en: http://www.dii.etsii.upm.es/aulaweb/informes.htm.

Bloom, B. S., Engelhart, M.D., Furst, E.J., Hill, W.K. y Krathwohl, D.R. Taxonomía de los Objetivos de la Educación, Tomo I, Ámbito del Conocimiento, Ed. Marfil, segunda edición, Alcoy, 1975.

Coronado, S. Garcia-Beltrán, A. Jaen, J. A. Martínez (1998), R. WebTutor, a knowlegde based system for evaluation and tutorship, Lecture Notes in Computer Science, Volume 1416, July 1998, Pages 727 - 734.

Ferre, M. Aracil, R. Sebastián, J. M. Pinto E. y Llorente J. Nueva metodología docente de las asignaturas de Regulación Automática I y Control por Computador, I Jornadas de Innovación Educativa de la Escuela Politécnica Superior de Zamora, Zamora (España), 20-22 de junio, 2006.

García, C. (2006) Evaluación en entornos virtuales de aprendizaje en Enseñanza y aprendizaje mediado por Internet: calidad de procesos y resultados, Cursos de verano 2006 de la Universidad Politécnica de Madrid.

García-Beltrán, A., Sánchez, M. J., Mira, J., y Martínez R. (1998), Análisis del Perfil de Conocimientos de Alumnos de Nuevo Ingreso en la Escuela Técnica Superior de Ingenieros Industriales de la Universidad Politécnica de Madrid, Enseñanza, 16, 23 39.

García-Beltrán, A. y Martínez, R. (2002) The Role of Self-Assesment in AulaWeb elearning System, 2002 EDEN - European Distance Education Network- Annual Conference, Granada (España), junio 16-19. 
García-Beltrán, A. y Martínez, R. (2004). Experiencia docente con AulaWeb en la asignatura de Informática, RED - Revista de Educación a Distancia, 11, (2004), Disponible en la URL: http://www.um.es/ead/red/11/REDAula.PDF.

Martínez, R. y García-Beltrán, A. (2001) AulaWeb: a WWW-Based Course-Support System with Self-Assessment and Student Tracking, World Conference on Educational Multimedia, Hypermedia and Telecommunications, ED-MEDIA 2001, Tampere, Finlandia.

Serrano, M. Torres, L.M. Pavón, I. y Sardá, E. (2004) Evalúe formativa y sumativamente en Capacidades docentes para una gestión de calidad en educación secundaria, Villar, L.M. (ed.), Mc. Graw-Hill, 259-269.

Villar, L.M. y Alegre O.M. (2004), Manual para la excelencia en la enseñanza superior, Mc Graw-Hill. 